\title{
Indikationen, chirurgische Technik und Ergebnisse der winkelstabilen Plattenosteosynthese bei periprothetischen Frakturen am Kniegelenk
}

\author{
Sven Märdian, Phillip Schwabe, Norbert P. Haas, Klaus-Dieter Schaser
}

\section{Zusammenfassung}

Die Inzidenz periprothetischer Frakturen des Kniegelenks (Femur, Tibia, Patella) steigt mit steigender Implantationsfrequenz von Knietotalendoprothesen stetig an. In Abhängigkeit von der Lokalisation können neben reinen Traumata auch implantatspezifische Parameter und Implantatlockerungen ursächlich sein. Es liegen inzwischen exakte Klassifikationen der periprothetischen Frakturen nach Knie-TEP für die 3 Lokalisationen an Femur, Tibia und Patella vor, die die Basis für ein differenziertes Therapiekonzept darstellen. Die modernen winkelstabilen Plattenfixateure als eine der vielfältigen Möglichkeiten verfügbarer Behandlungsoptionen stellen eine valide Therapieoption dar. Trotzdem weisen die Behandlungsergebnisse hohe Raten an Komplikationen auf, was nur durch die adäquate Analyse der Frakturursachen und die Umsetzung in ein individuell optimiertes, weichteilschonendes Behandlungskonzept $\mathrm{zu}$ beheben ist. Hierdurch kann eine vergleichsweise identische funktionelle Wiederherstellung des Patienten wie vor dem Frakturereignis erzielt werden.
Indications, Surgical Technique and Outcome of Angular Stable Plates in Periprosthetic Fractures of the Knee

The incidence of periprosthetic fractures of the knee (femur, tibia, patella) increases with the increasing frequency of total arthroplasties of the knee. Depending on the localisation the causes can be not only purely traumatic but also due to implant-specific parameters and implant loosenings. In the meantime there is an exact classification of the periprosthetic fractures after total knee arthroplasty for the three localisations on the femur, tibia, and patella that provides a basis for differentiated therapeutic concepts. Modern, angular stable plate fixation methods, as one of the many treatment options, represent a valid therapeutic choice. Even so, the treatment results exhibit a high rate of complications that can only be avoided by an adequate analysis of the fracture cause and the application of an individually optimised, soft-tissue sparing treatment concept. In this way a more or less complete restoration of the functional state existing prior to the fracture can be achieved. differentes Therapiekonzept. Da die periprothetischen Frakturmuster sich nicht den typischen Frakturklassifikationen der allgemeinen Traumatologie zuordnen lassen, sind diese klassischen Frakturklassifikationen auch nicht hilfreich und können nicht als Grundlage für die Therapieentscheidung periprothetischer Frakturen dienen [6]. Durch eine konsequente Systematisierung wurden viele Klassifikationen periprothetischer Frakturen erarbeitet.

In den letzten Jahren haben sich die Klassifikationen von Rorabeck et al. sowie Su et al. für periprothetische Femurfrakturen, die Klassifikation von Felix et al. für tibiale Frakturen sowie die Klassifikation von Goldberg et al. für die Patellafrakturen durchgesetzt (vgl. Tab. 1, 2 und 3) [2,7-9].

Auf der Basis dieser Klassifikationen können die Differenzialindikationen der Rekonstruktionsverfahren bestimmt und korrespondierenden Therapiealgorithmen zugeordnet werden (Abb. 1 und 2), wobei die Versorgung mit winkelstabilen Plattensystemen den Femur- und Tibiafrakturen vorbehalten ist.

Analog zur Versorgung von periprothetischen Frakturen an der Hüfte ist die zentrale Frage jeglicher Therapieentscheidung, ob die Prothese gelockert ist oder nicht.

Diese zentrale Frage stellt letztlich Drehund Angelpunkt in der Wahl des einzusetzenden operativen Verfahrens (osteosynthetische Rekonstruktion versus Komponenten-/Prothesenwechsel) dar [6]. In den meisten Fällen resultieren periprothetische Frakturen postoperativ aufgrund eines Niedrigenergietraumas, seltener als Folge von Hochrasanztraumata, welche dann meist im Rahmen von Verkehrsunfällen auftreten. Stressoder Ermüdungsfrakturen sind meist Folge von Fehlimplantationen (femorales notching) sowie septischen oder aseptischen Prothesenlockerungen [10-12].
OP-JOURNAL 2012; 28: 160-165

(C) Georg Thieme Verlag KG Stuttgart · New York DOI http://dx.doi.org/10.1055/s-0032-1315337 totalendoprothesen bedingen die Unterschiede in der anatomischen Region sowie implantatassoziierte Konstruktionsmerkmale der Knieendoprothetik ein 
Tab. 1 Klassifikation der periprothetischen Femurfraktur nach Rorabeck und Su.

\begin{tabular}{lll} 
& Rorabeck-Klassifikation & Su-Klassifikation \\
\hline Typ I & $\begin{array}{l}\text { nicht dislozierte Fraktur bei fester } \\
\text { Prothese }\end{array}$ & $\begin{array}{l}\text { Fraktur liegt proximal der femoralen } \\
\text { Prothesenkomponente }\end{array}$ \\
\hline Typ II & $\begin{array}{l}\text { dislozierte Fraktur bei fester } \\
\text { Prothese }\end{array}$ & $\begin{array}{l}\text { Fraktur beginnt auf Höhe des femoralen } \\
\text { Schildes und zieht nach proximal }\end{array}$ \\
\hline Typ III & $\begin{array}{l}\text { Prothese locker, Fraktur kann dis- } \\
\text { loziert oder nicht disloziert sein }\end{array}$ & $\begin{array}{l}\text { sämtliche Frakturanteile liegen distal } \\
\text { des anterioren Prothesenschilds }\end{array}$
\end{tabular}

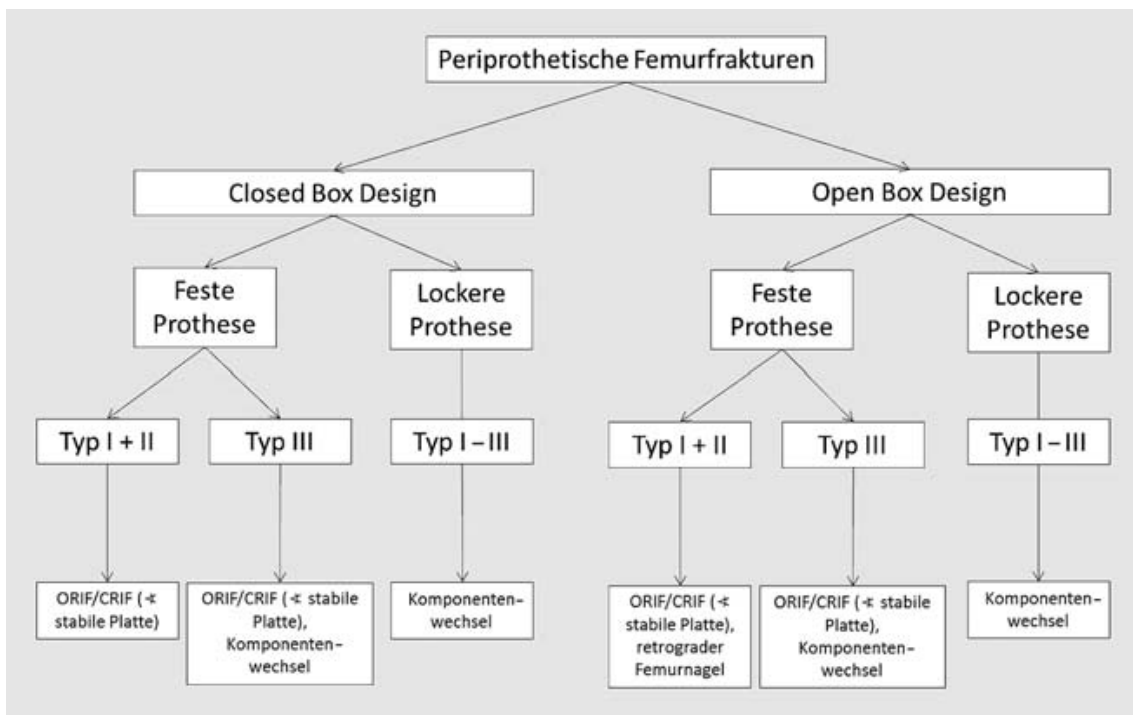

Abb. 1 Therapiealgorithmus periprothetischer Femurfrakturen (modifiziert nach [1]).

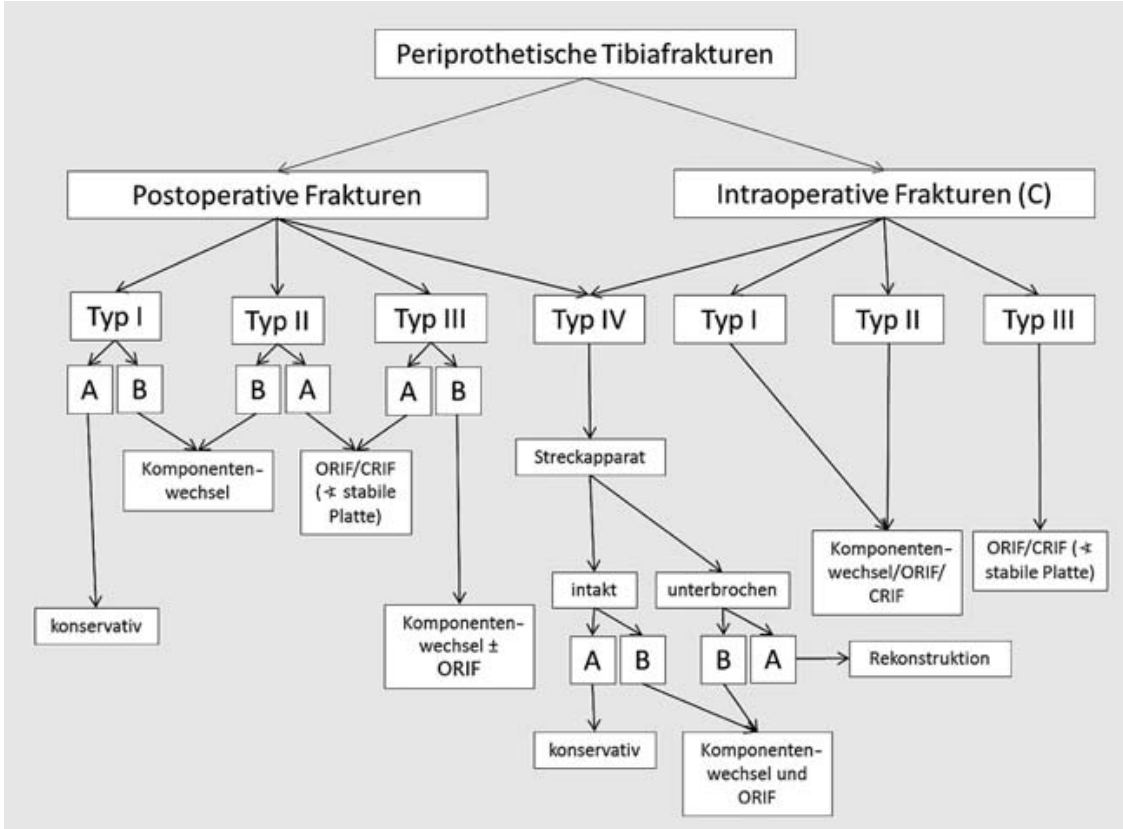

Abb. 2 Therapiealgorithmus periprothetischer Tibiafrakturen (modifiziert nach [1]).

Da diese Frakturen meist in einem älteren Patientenklientel mit vielen Komorbiditäten gesehen werden, ist die operative Versorgung dieser Frakturen bei meist osteoporotischem Knochen eine große chirurgische Herausforderung. Diese fordert dem Unfallchirurgen und Orthopäden einen großen Erfahrungsschatz an biomechanischem Wissen und operativer Erfahrung ab, als auch Kennt-
Tab.2 Klassifikation der periprothetischen Tibiafraktur nach Felix.

\begin{tabular}{ll} 
& Felix-Klassifikation \\
\hline Typ I & $\begin{array}{l}\text { liegen im Bereich des Tibiakopfs } \\
\text { und ziehen in das Interface }\end{array}$ \\
\hline Typ II & $\begin{array}{l}\text { liegen im meta-/diaphysären } \\
\text { Übergangsbereich }\end{array}$ \\
\hline Typ III & $\begin{array}{l}\text { liegen unterhalb der } \\
\text { Tibiakomponente }\end{array}$ \\
\hline Typ IV & Frakturen der Tuberositas tibiae \\
\hline Subtyp A & Prothese stabil \\
\hline Subtyp B & Prothese locker \\
\hline Subtyp C & intraoperative Fraktur
\end{tabular}

Tab.3 Klassifikation der periprothetischer Patellafraktur nach Goldberg.

\begin{tabular}{cl} 
& Klassifikation nach Goldberg \\
\hline Typ I & $\begin{array}{l}\text { Streckapparat intakt, Patella- } \\
\text { implantat stabil, vor allem } \\
\text { periphere Frakturen }\end{array}$ \\
\hline Typ II & $\begin{array}{l}\text { entweder Streckapparat oder } \\
\text { Implantatlager beschädigt }\end{array}$ \\
\hline Typ III & $\begin{array}{l}\text { A: mit Läsion des Lig. patellae } \\
\text { (Streckapparat beschädigt) } \\
\text { B: keine Dislokation (Streck- } \\
\text { apparat intakt) }\end{array}$ \\
\hline Typ IV & $\begin{array}{l}\text { sämtliche Formen in Kom- } \\
\text { bination mit einer Luxation }\end{array}$
\end{tabular}

nisse und Fertigkeiten in verschiedensten Osteosynthesetechniken. Zudem ist die Beherrschung des gesamten Spektrums der Revisionsendoprothetik notwendig, um im Salvagefall Rückzugsoptionen vorzuhalten.

\section{Ursachen, Risikofaktoren und Epidemiologie}

Viele Autoren waren in der Vergangenheit in der Lage, wichtige patientenspezifische Risikofaktoren zu identifizieren, welche das Auftreten periprothetischer Frakturen begünstigen. Unter anderem konnten die Osteoporose [13-15], die rheumatoide Arthritis [14,16-18], lange Steroid- oder Kortikoidtherapie [17-20], vorbestehende neurologische Erkrankungen wie z. B. zerebrale Ataxie, Morbus Parkinson, Myasthenia gravis und viele andere [16], bereits vorhandene Revisionsprothesen $[13,14]$, Arthrofibrose (aufgrund reduzierter Flexion im Knie und damit einhergehender höherer Hebelkräfte beim Gehen) [21] als auch progrediente lokale Osteolysen auf dem Bo- 
den septischer oder aseptischer Lockerungsvorgänge [10-12] als solche erkannt werden. Aktuell wird die Inzidenz periprothetischer Frakturen nach totalem Kniegelenksersatz mit etwa $1 \%(0,3-$ $2,5 \%$ ) angegeben $[7,21]$. Periprothetische Femurfrakturen finden sich mit 0,3-2\% häufiger als periprothetische Frakturen der Tibia mit $0,3-0,5 \%$ oder der Patella mit bis zu 0,6\% [22,23]. Erwartungsgemäß ist bei und nach einem Prothesenwechsel mit einer höheren Inzidenz periprothetischer Frakturen zu rechnen als im Rahmen der Primärendoprothetik [7].

\section{Diagnostik}

Zur Diagnosefindung sind eine ausführliche Anamnese und klinische Untersuchung (neurovaskulärer Status, Weichteilschaden, laborchemische Entzündungsparameter) insofern von enormer Bedeutung, als dass potenzielle klinische Zeichen einer vorbestehenden Prothesenlockerung (Belastungs- und Bewegungsschmerzen, Gehstrecke, Instabilität und Fehlstellung) eruiert werden können. Die subjektiven Angaben des Patienten in Zusammenschau mit den objektiv zu erhebenden Befunden und Funktionseinschränkungen sind in Anbetracht der manchmal schwierigen Interpretation der radiologischen Diagnostik (lockere oder feste Prothesenverankerung) oftmals das einzige Kriterium einer Prothesenlockerung [6].

Eine konventionelle Röntgenaufnahme des Kniegelenks in 2 Ebenen, welche das einliegende Prothesenimplantat vollständig abbildet, ist essenziell.

Im Falle von ebenfalls vorhandenen endoprothetischen Gelenkersätzen der benachbarten Gelenke oder vorangegangener Frakturen (Femur, Tibia, Hüfte, OSG) ist eine vollständige Abbildung des frakturierten Skelettabschnitts notwendig, um die dort einliegenden Implantate und deren Lage zur Fraktur beurteilen zu können. Grundsätzlich sollte femoral wie tibial die gesamte Strecke, welche bspw. durch langstreckige Implantate überbrückt werden soll, biplanar abgebildet werden. Optional muss bei Verdacht auf Verletzung des Kniestreckapparats eine axiale Aufnahme der Patella erfolgen, um Frakturen/Subluxationen zu erkennen. Um die Frage der Prothesenlockerung suffizienter zu beantworten, ist es hilfreich, Voraufnahmen zu beurteilen und sich den Verlauf vor Augen zu führen, um ggf. progrediente Osteolysen zu erkennen. Weiterführende CT-

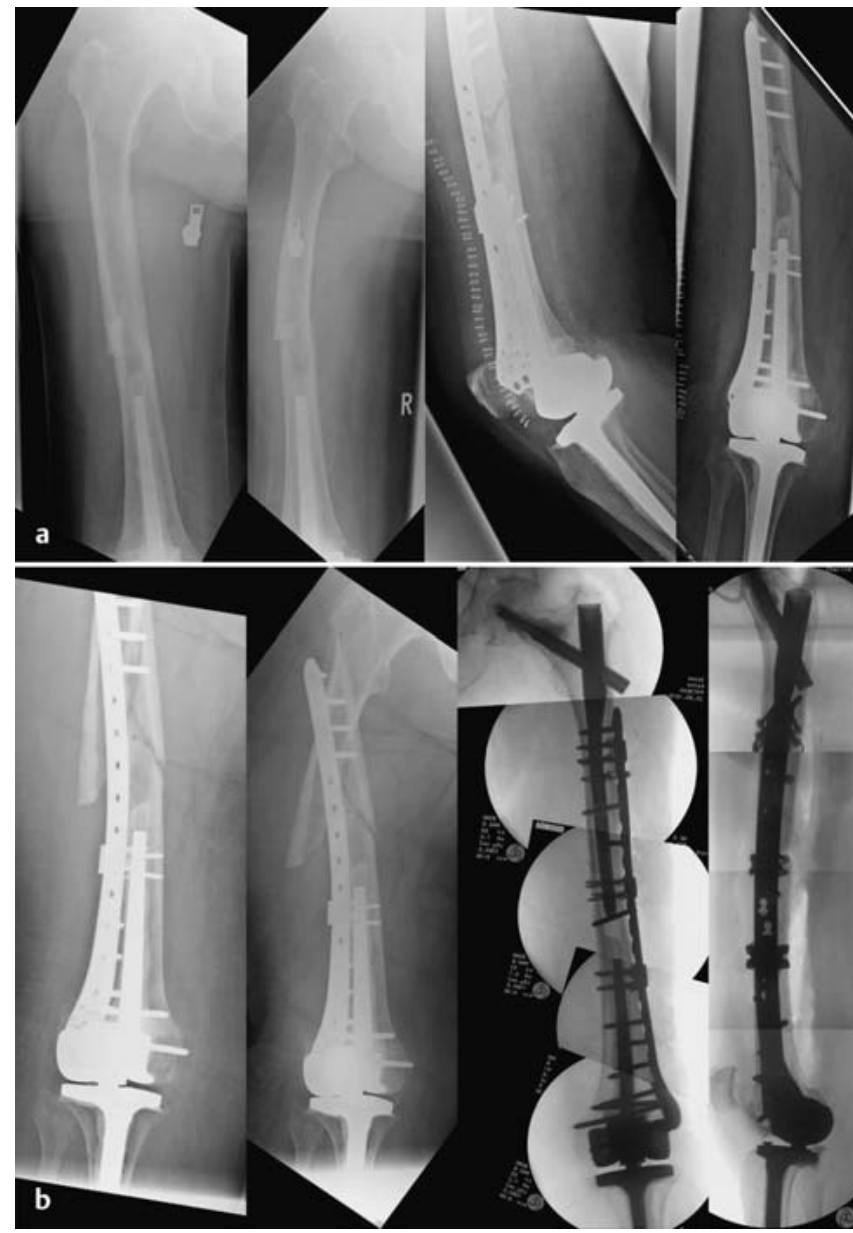

Abb. $3 \mathbf{a}$ und $\mathbf{b}$ a 93 J., weiblich, Sturz im häuslichen Umfeld, Typ-I-Fraktur nach Su (Versorgung mit L.I.S.S.-Plattenfixateur, Firma Synthes). $\mathbf{b}$ Erneuter Sturz, Revisionseingriff bei periimplantärer Fraktur (zementierter PFNa, VA-Kondylenplatte Femur, Locking Attachment Plates, F. Synthes).
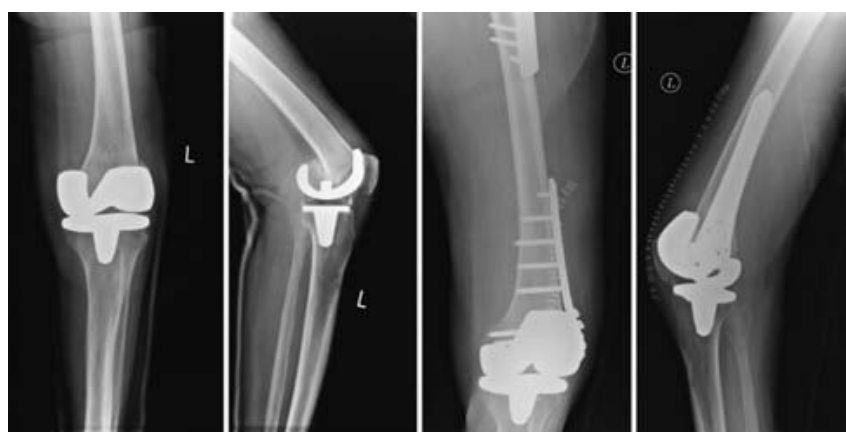

Abb. 4 86 J. nach häuslichem Sturz, Typ-III-Fraktur nach Su.

Diagnostik ist trotz entstehender Metallartefakte immer dann erforderlich und hilfreich, wenn wenig dislozierte Frakturen in der konventionell radiologischen Diagnostik nicht suffizient verfolgt oder ausgeschlossen werden können und wenn der Frakturverlauf durch die Prothesenkomponenten selbst (Prothesenschild, vorhandene Schäfte) verdeckt wird.

Die MRT-Diagnostik hat aufgrund erheblicher Auslöschungsartefakte durch das Implantat keinen Stellenwert in der Diagnostik periprothetischer Frakturen [6].

\section{Therapie}

Die allgemeinen Behandlungsziele bestehen in einer raschen Frakturheilung (binnen 6 Monate), Wiederherstellung der anatomiegerechten Achs- und Torsionsverhältnisse (Varus-/Valgusfehler $<5^{\circ}$, Ante-/Retroflexion $<10^{\circ}$, Verkürzung $<1 \mathrm{~cm}$ ), Erreichen einer schmerzfreien Funktion und Beweglichkeit wie vor der Fraktur (Minimum Range of Motion von $90^{\circ}$ ) $[24,25]$. Die Ausheilung der periprothetischen Fraktur in Fehlstellung bedingt zwangsläufig eine Mehrbelastung der Prothese und geht mit einer höheren Lockerungsrate einher [7]. Die konservative Therapie periprotheti- 


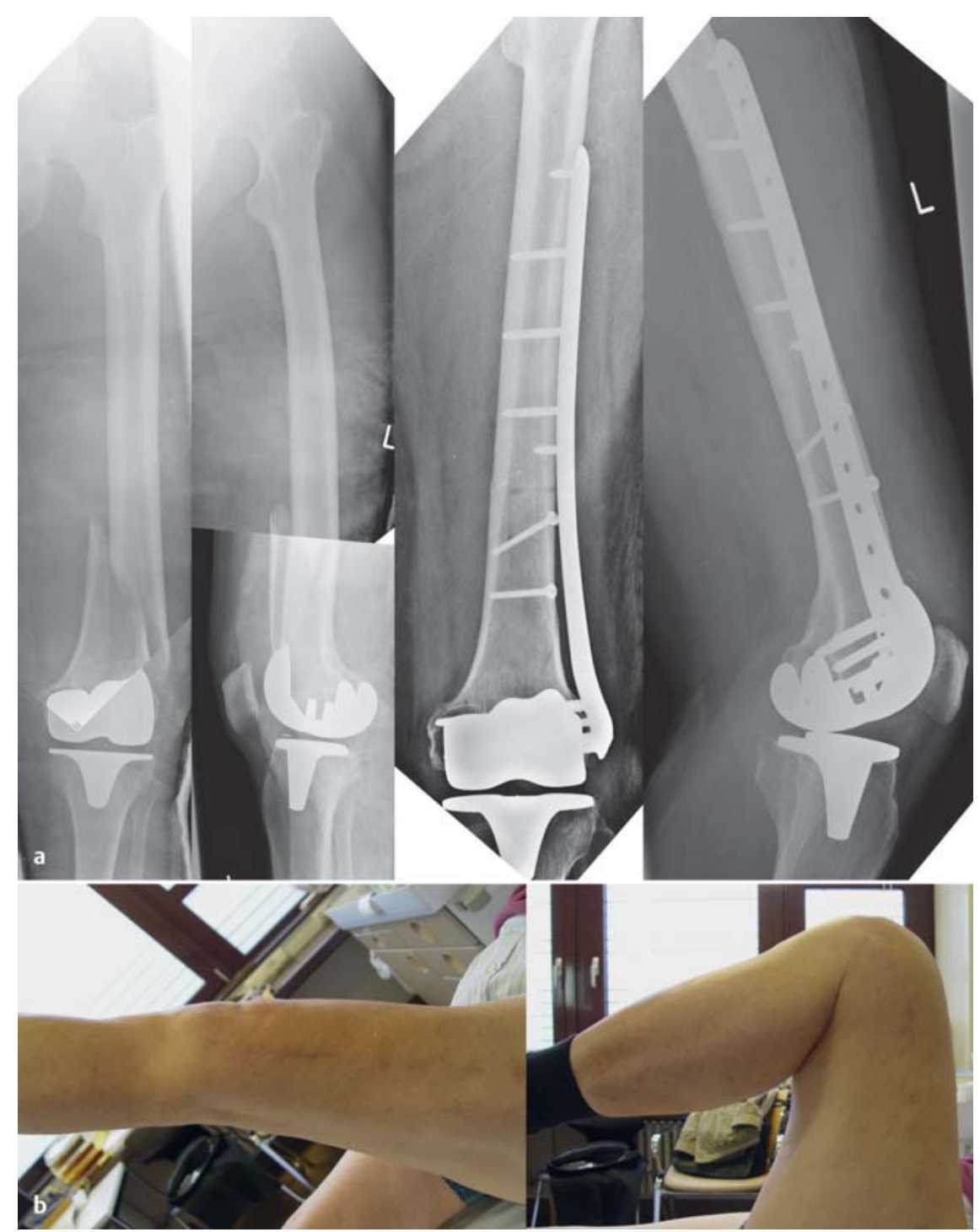

scher Frakturen am Knie kommt aufgrund der o.g. Behandlungsziele nur für stabile, nicht dislozierte Frakturen in Betracht, wie z.B. Ermüdungsfrakturen [6].

Vor jeglicher operativer Intervention bedarf es einer guten präoperativen Planung, welche auf der Basis von

- patientenspezifischen Charakteristika (Funktion, Operabilität, Zusatzmorbiditäten),

- einer korrekten Frakturklassifikation mit Einschätzung der Festigkeit der Prothese,

- einer genauen Analyse der Fraktur sowie des einliegenden Prothesentyps/ -designs (open-/closed box, zementiert/zementfrei, mit/ohne Schaftverlängerung, not constrained/semiconstrained/constrained, Verfügbarkeit sämtlicher erforderlicher Instrumentarien [ggf. Leihinstrumentarien] und Prothesenimplantate, Vorhandensein von Revisionsimplantaten im Falle eines notwendigen Prothesenwechsels) konzipiert werden muss.
Grundvoraussetzung für die osteosynthetische Rekonstruktion ist die feste Prothese. Gelockerte Prothesen erfordern den Prothesen- oder Komponentenwechsel. Dennoch sind, auch bei mutmaßlich stabilen Prothesenverhältnissen, extra- wie intramedulläre Osteosyntheseverfahren bis hin zur Möglichkeit des Prothesenwechsels vorzuhalten, um intraoperativ auf unvorhergesehene Änderungen (wider Erwarten lockere Prothesenkomponenten, Ausdehnung der Frakturzone an die knöcherne Verankerung der Prothese usw.) adäquat reagieren zu können.

\section{Konservative Therapie}

Die konservative Therapie der periprothetischen Frakturen im Bereich des Kniegelenks beschränken sich heute nur noch auf stabile Ermüdungsfrakturen [1], da sonst die o.g. Behandlungsziele nicht erreicht werden können.
Abb. 5 a und b a Typ-II-Fraktur nach Su, perkutane Zugschraubenosteosynthese in Kombination mit minimalinvasiver L. I.S.S.Plattenosteosynthese (Firma Synthes). b Klinisches Ergebnis 6 Monate postoperativ.

\section{Planung und Timing}

Für eine erfolgreiche osteosynthetische Versorgung ist eine genaue präoperative Planung unumgänglich. Hierzu ist zwingend eine präoperative Skizze mit Implantatwahl, -platzierung sowie der Schraubenbelegung (mono-/bikortikal, periprothetische Kurzschrauben im Schaftbereich der Prothese) anzufertigen. Im Falle von Langschaftprothesen sind ggf. polyaxiale Implantate (NCB Firma Zimmer, VA-LCP Kondylenplatte Firma Synthes) oder aber zusätzliche „locking attachment plates“ oder Cerclagensysteme erforderlich, deren Platzierung ebenfalls sorgfältig und biomechanisch sinnvoll geplant werden muss. Im Falle von großen Trümmerzonen ist über zusätzliche biologische Adjuvantien (Spongiosaplastik, Beckenkammspan, Wachstumsfaktoren, Strut Graft) zu diskutieren. Langstreckige, suffizient fixierte Plattenfixateursysteme mit frakturnahen unbesetzten Plattenlöchern sind dabei als günstiger anzusehen, vermeiden erhöhten Stress am Implantat und reduzieren die Rate von Implantatversagern. Für interprothetische/periimplantäre Frakturen bedeutet dies auch, dass z. B. extramedulläre Plattenfixateursysteme die intramedullären Schaft-/Implantatanteile, wie z.B. die Hüft-TEP-Schaftspitze, überragen sollten (Abb. $\mathbf{3}$ a und $\mathbf{b}$ ).

\section{Periprothetische Femurfrakturen}

Die periprothetische Femurfraktur ist mit 0,3-2,5\% die größte Subgruppe der periprothetischen Frakturen nach erfolgter Knie-Totalendoprothese.

Abgesehen von den genannten allgemeinen Risikofaktoren, welche eine solche Fraktur, begünstigen bestehen spezielle Risikofaktoren wie z.B. das femorale „notching“ während der Primärimplantation. Biomechanische Studien konnten zeigen, dass dieses Notching den anterioren Kortex deutlich schwächt und somit die Entstehung einer postoperativen Fraktur begünstigt $[13,26]$. Eine korrekte präoperative Klassifikation der Fraktur und damit einhergehend der korrekte Therapiealgorithmus, Kenntnis des einliegenden Prothesentyps und chirurgische Expertise sind unabdingbare Voraussetzung für eine erfolgreiche $\mathrm{Be}$ - 


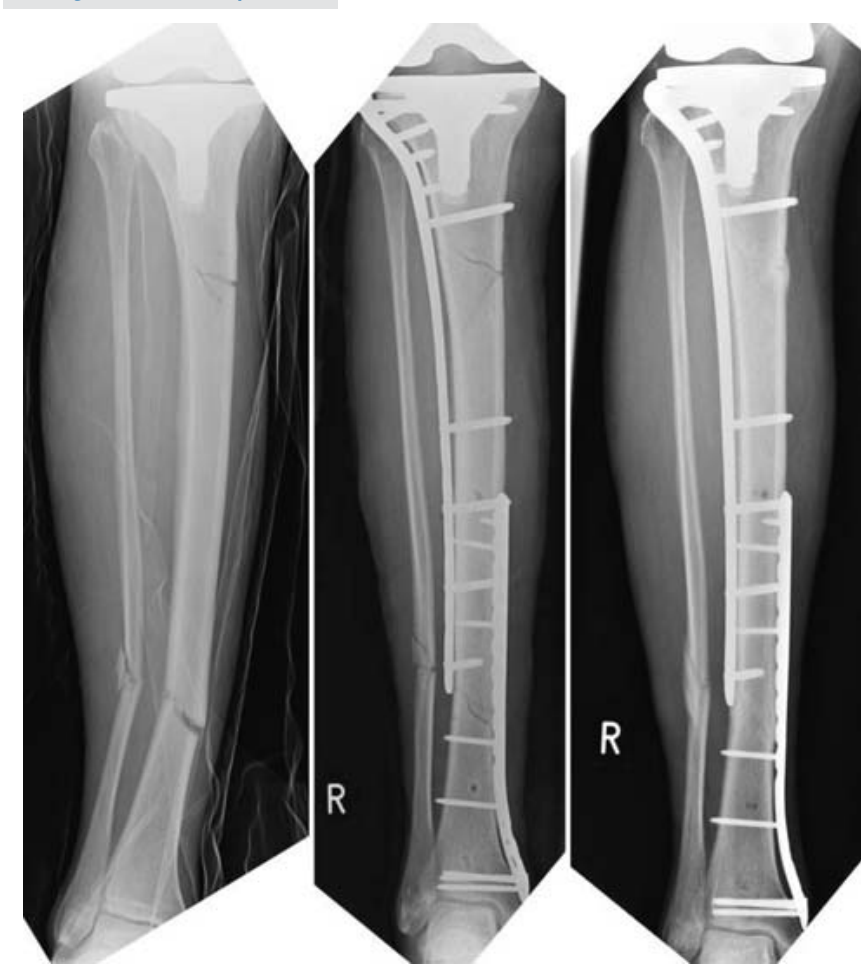

Abb. 6 Typ-III-Fraktur nach Felix, minimalinvasive Plattenosteosynthese (L.I.S.S., low bend LCP, Firma Synthes).

handlung des oft betagten und multimorbiden Patienten.

Die Domäne der winkelstabilen Plattenosteosynthese sind dabei in erster Linie die Typ-I- und Typ-II-Frakturen (nach Su).

Im Falle von Typ-III-Frakturen ist eine suffiziente distale Frakturverankerung das maßgebliche Problem, sodass hier bei genügendem Knochenlager nach distal die winkelstabile Plattenosteosynthese (Abb. 4), bei ungenügendem Knochenlager nur der Prothesenwechsel infrage kommt. Die modernen winkelstabilen Fixateursysteme erlauben selbst im osteoporotischen Knochen einen optimalen Halt und langfristig reduziertes Lockerungsverhalten. Durch die erhaltene periostale Durchblutung wird eine optimale Frakturheilung gewährleistet. Zusätzlich kann durch das LCP-Prinzip, welches das wahlweise Einbringen konventioneller Kortikalis-/Spongiosaschrauben erlaubt, interfragmentäre Kompression im klassischen Sinne aufgebaut werden.

Die Prinzipien der biologischen Osteosynthese können mit limitiertem $\mathrm{Zu}$ gang, indirekter Reposition und perkutaner Fixierung der Schrauben im Sinne eines minimalinvasiven Konzepts umgesetzt werden und somit essenziell zur Schonung der kniegelenksnahen Weichteile beitragen [1] (Abb. 5a und b).

\section{Periprothetische Tibiafrakturen}

Die periprothetische Fraktur der Tibia ist in ihrer Inzidenz von 0,3-0,5\% aller implantierten Knie-Totalendoprothesen signifikant seltener als die des Femurs [23].

Als spezielle Risikofaktoren konnten bisher Varus-/Valgusfehlstellungen sowie Fehlrotationen der tibialen Komponente, forcierte Impaktionen im Rahmen der Implantation der Komponente sowie progrediente Osteolysen identifiziert werden [1,23]. Analog zur Versorgung der periprothetischen Femurfraktur gelten die akribische Klassifikation und Planung als Grundvoraussetzung der erfolgreichen Osteosynthese.

Vor allem die Typ-II und Typ-III (nach Felix)-Frakturen gelten als Domäne der winkelstabilen Plattenosteosynthese.

Auch hier sind die Prinzipien der biologischen Osteosynthese einzuhalten, um v.a. beim beschränkten Weichteilmantel der Tibia eine ungestörte Frakturheilung zu gewährleisten (Abb.6). Im Falle der periprothetischen Tibiafraktur sollten die o.g. speziellen Risikofaktoren unbedingt überprüft werden, um einen nachhaltigen Therapieerfolg zu haben und ggf. z.B. bei ausgeprägter Malrotation dem Komponentenwechsel den Vorzug vor der Rekonstruktion zu geben.

\section{Komplikationen}

Die Komplikationsraten sind je nach Frakturtyp, Ausmaß der zugrunde liegenden Osteoporose und verwendetem Implantat unterschiedlich hoch.

Die periprothetischen Tibiafrakturen haben dabei eine deutlich höhere Rate als korrespondierende Frakturen am Femur.

$\mathrm{Zu}$ beobachten sind verzögerte Durchbauung/Pseudarthrose, Fehlstellungen, Refrakturen, tiefe Infekte, Arthofibrosen und Implantatversagen [1]. Es ist zu erwarten, dass durch die zunehmende Anwendung winkelstabiler Implantate eine Reduktion zumindest der mechanisch induzierten Komplikationen zu erzielen ist.

\section{Fazit für die Praxis}

Periprothetische Frakturen nach KnieTEP haben eine steigende Inzidenz. Bei stabiler Prothese sollte die osteosynthetische Rekonstruktion zumeist mit winkelstabilen Plattenfixateursystemen vorgenommen werden. Funktionell entscheidend ist auch eine geringe Kompromittierung der kniegelenksnahen Weichteile. Lockere Prothesen benötigen den Prothesenwechsel der Komponente nach allen Regeln der Revisionsendoprothetik am Kniegelenk. Vorausgehen muss jedoch immer eine exakte Analyse der Fraktur, korrekte Klassifikation und sorgfältige Planung, um annäherungsweise wieder den funktionellen Status wie vor der Fraktur zu erreichen.

\section{Literatur}

${ }^{1}$ Mittlmeier $T$ et al. [Periprosthetic fractures after total knee joint arthroplasty]. Unfallchirurg 2005; 108: 481-495

2 Felix NA, Stuart MJ, Hanssen AD. Periprosthetic fractures of the tibia associated with total knee arthroplasty. Clin Orthop Relat Res 1997; 345: 113-124

${ }^{3}$ Figgie MP, et al. The results of treatment of supracondylar fracture above total knee arthroplasty. J Arthroplasty 1990; 5: 267-276

${ }^{4}$ Babis GC et al. High energy tibial plateau fractures treated with hybrid external fixation. J Orthop Surg Res 2011; 6: 35

5 Rorabeck CH, Taylor JW. Periprosthetic fractures of the femur complicating total knee arthroplasty. Orthop Clin North Am 1999; 30: 265-277

${ }^{6}$ Chmell MJ, Moran MC, Scott RD. Periarticular fractures after total knee arthroplasty: principles of management. J Am Acad Orthop Surg 1996; 4: 109-116

${ }^{7}$ Su ET, DeWal H, Di Cesare PE. Periprosthetic femoral fractures above total knee replacements. J Am Acad Orthop Surg 2004; 12: $12-$ 20 
${ }^{8}$ Rorabeck CH, Taylor JW. Classification of periprosthetic fractures complicating total knee arthroplasty. Orthop Clin North Am 1999; 30: 209-214

${ }^{9}$ Goldberg VM et al. Patellar fracture type and prognosis in condylar total knee arthroplasty. Clin Orthop Relat Res 1988: 115-122

10 Rand JA. Supracondylar fracture of the femur associated with polyethylene wear after total knee arthroplasty. A case report. J Bone Joint Surg [Am] 1994; 76: 1389-1393

${ }^{11}$ Rand JA, Coventry MB. Stress fractures after total knee arthroplasty. J Bone Joint Surg [Am] 1980; 62: 226-233

12 Scott RD, Turoff N, Ewald FC. Stress fracture of the patella following duopatellar total knee arthroplasty with patellar resurfacing. Clin Orthop Relat Res 1982; 170: 147-151

${ }^{13}$ Cain PR et al. Periprosthetic femoral fractures following total knee arthroplasty. Clin Orthop Relat Res 1986; 208: 205-214

${ }^{14}$ Merkel KD, Johnson jr EW. Supracondylar fracture of the femur after total knee arthroplasty. J Bone Joint Surg [Am] 1986; 68: 29-43

15 Shawen SB et al. Osteoporosis and anterior femoral notching in periprosthetic supracondylar femoral fractures: a biomechanical analysis. J Bone Joint Surg [Am] 2003; 85: 115-121

${ }^{16}$ Culp RW et al. Supracondylar fracture of the femur following prosthetic knee arthroplasty. Clin Orthop Relat Res 1987; 222: 212-222
${ }^{17}$ McLaren AC, Dupont JA, Schroeber DC. Open reduction internal fixation of supracondylar fractures above total knee arthroplasties using the intramedullary supracondylar rod. Clin Orthop Relat Res 1994; 302: 194-198

18 Sisto DJ, Lachiewicz PF, Insall JN. Treatment of supracondylar fractures following prosthetic arthroplasty of the knee. Clin Orthop Relat Res 1985; 196: 265-272

${ }^{19}$ Herrera DA et al. Treatment of acute distal femur fractures above a total knee arthroplasty: systematic review of 415 cases (19812006). Acta Orthop 2008; 79: 22-27

${ }^{20}$ Hirsh DM, Bhalla S, Roffman M. Supracondylar fracture of the femur following total knee replacement. Report of four cases. J Bone Joint Surg [Am] 1981; 63: 162-163

${ }^{21}$ Ayers DC. Supracondylar fracture of the distal femur proximal to a total knee replacement. Instr Course Lect 1997; 46: 197-203

22 Berry DJ. Patellar fracture following total knee arthroplasty. J Knee Surg 2003; 16: 236-241

23 Burnett RS, Bourne RB. Periprosthetic fractures of the tibia and patella in total knee arthroplasty. Instr Course Lect 2004; 53: 217235

${ }^{24}$ DiGioia 3rd AM, Rubash HE. Periprosthetic fractures of the femur after total knee arthroplasty. A literature review and treatment algorithm. Clin Orthop Relat Res 1991; 271: 135-142
${ }^{25}$ Healy WL, Siliski JM, Incavo SJ. Operative treatment of distal femoral fractures proximal to total knee replacements. J Bone Joint Surg [Am] 1993; 75: 27-34

${ }^{26}$ Lesh ML et al. The consequences of anterior femoral notching in total knee arthroplasty. A biomechanical study. J Bone Joint Surg [Am] 2000; 82: 1096-1101

\section{Dr. med. Sven Märdian}

Oberarzt

Dr. med. Phillip Schwabe

Oberarzt

Univ.-Prof. Dr. Dr. h.c.

Norbert P. Haas

Klinikdirektor

Univ.-Prof. Dr. med.

Klaus-Dieter Schaser

stellvertretender Kliniksdirektor

CMSC - Centrum für Muskuloskeletale Chirurgie

Charité - Universitätsmedizin Berlin

Augustenburger Platz 1

13353 Berlin

sven.maerdian@charite.de 